Virtual photons, dipole fields and energy transfer: a quantum electrodynamical approach

This content has been downloaded from IOPscience. Please scroll down to see the full text. 2004 Eur. J. Phys. 25845

(http://iopscience.iop.org/0143-0807/25/6/017)

View the table of contents for this issue, or go to the journal homepage for more

Download details:

IP Address: 139.222.115.136

This content was downloaded on 13/11/2014 at 12:34

Please note that terms and conditions apply. 


\title{
Virtual photons, dipole fields and energy transfer: a quantum electrodynamical approach
}

\author{
David L Andrews and David S Bradshaw \\ Nanostructures and Photomolecular Systems, School of Chemical Sciences, \\ University of East Anglia, Norwich, NR4 7TJ, UK \\ E-mail: david.andrews@physics.org
}

Received 25 August 2004, in final form 2 September 2004

Published 30 September 2004

Online at stacks.iop.org/EJP/25/845

doi:10.1088/0143-0807/25/6/017

\begin{abstract}
Dipole emission mechanisms for energy transfer operate in many important areas of photophysics. A straightforward analysis based on quantum electrodynamics not only reveals the entanglement of mechanisms usually regarded as 'radiative' and 'radiationless'; it also gives significant physical insights into a host of topics in electromagnetism. These include: the designation of real and virtual photons; propagating and non-propagating character in electromagnetic fields; near-zone and wave-zone effects; transverse and longitudinal character; the effects of retardation; the relation between couplings of static and transition dipoles, and manifestations of quantum uncertainty. A simple extension of the theory to accommodate magnetic dipole as well as electric dipole transitions furthermore reveals key differences between the range dependences of the magnetic and electric fields produced by dipolar emission. With important technological applications, this lesson in advanced physics showpieces the interplay of principles associated with quantum mechanics, electromagnetism and photophysics.
\end{abstract}

\section{Introduction}

The migration of energy from electronically excited atomic or molecular donors to acceptors in the electronic ground state is a mechanism that plays a key role in a wide range of optical and photophysical phenomena. Important examples include: natural light-harvesting [1, 2]especially energy hopping between chlorophyll molecules in the photosynthetic unit; lightharvesting in synthetic polymers [3, 4]—-materials that mimic biological antenna complexes; methods for nanoscale structure determination $[5,6] —$ such as the use of the popularly termed 'spectroscopic ruler' to deliver information on intramolecular distances in proteins, and 


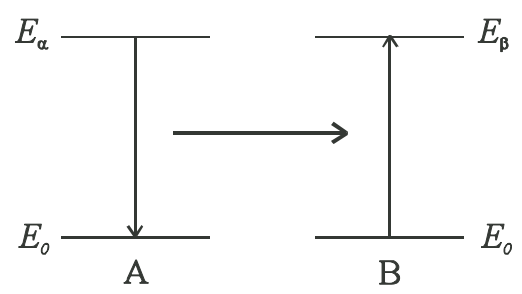

Figure 1. Energy-level diagram for resonance energy transfer: $E_{0}$ represents the ground-state energy; $E_{\alpha}$ and $E_{\beta}$ denote the excited state energies of donor A and acceptor B, respectively. Vertical arrows represent electronic transitions and the horizontal arrow denotes the energy transfer.

nonlinear optics-particularly frequency conversion in materials such as lanthanide-doped crystals [7, 8]. Despite its fundamental nature, energy transfer over any distance beyond wavefunction overlap-i.e. from nanoscale to arbitrarily large donor-acceptor separationsentails a resonance energy transfer (RET) mechanism whose correct description can only be derived using the principles of quantum electrodynamics. QED tells us that each such excitation transfer is mediated by a virtual photon, a messenger particle that cannot be directly detected and which, by its creation and subsequent annihilation, electromagnetically couples the donor decay and acceptor excitation. An energy-level depiction of the transfer between a donor atom $\mathrm{A}$ and an acceptor B is shown in figure 1.

As well as having major photophysical and technological applications, the theory of resonance energy transfer affords significant physical insights into a host of topics in electromagnetism and quantum mechanics, and this is the main motivation for the present analysis. Current interest in the didactic value of such topics is also illustrated by recent work on the principle of causality in electromagnetic theory [9], the status of relativistic fields [10], fields associated with a moving point charge [11], and the relationship between classical and quantum electrodynamical radiation formulae [12]. Since RET is mainly associated with E1-E1 dynamic coupling, i.e. electric dipole transitions in both donor and acceptor, we begin in section 2 with a QED derivation of the E1-E1 coupling tensor. The ensuing discussion also includes a comparison of dynamic with the more familiar static (conventional dipole-dipole) coupling. In certain cases, where the donor or acceptor transition is electric dipole forbidden on symmetry grounds but allowed through magnetic dipole (M1) coupling, E1-M1 coupling becomes the principal transfer mechanism [13]; accordingly the E1-M1 coupling tensor is derived and examined in section 3. It is our intention to cultivate facility with the calculational methods and also to elicit fresh insights into a number of physical features, including the fundamental nature of the electromagnetic mediation of energy transfer. Where appropriate, instructive comparisons are drawn with the corresponding classical expressions - though it may be noted that the classical interpretation furnishes less physical information on the electromagnetic fields; this represents a further justification for the detailed QED formulation. We conclude with a general discussion in section 4 .

\section{E1-E1 coupling}

\subsection{Derivation of the coupling tensor}

Unlike classical electrodynamics, where radiation is treated in terms of waves and charges act as particles in fields determined by Maxwell's equations, a QED framework treats both fields and matter on the same quantum basis. The detail which emerges from the theory to be developed below itself represents a compelling case for a QED analysis; however we 
assume that students of the subject will have already learned about the broader successes and applications of the theory such as its prediction of the Casimir effect [14].

As a starting point for the analysis, the non-relativistic Hamiltonian energy of a system comprising atoms or molecules labelled $\xi$ is promoted to operator status producing an operator, $H$, which in multipolar form is exactly expressible as

$$
H=\sum_{\xi} H_{\text {mat }}(\xi)+\sum_{\xi} H_{\text {int }}(\xi)+H_{\text {rad }},
$$

where $H_{\text {mat }}$ is the matter Hamiltonian, $H_{\text {rad }}$ is the radiation Hamiltonian and $H_{\text {int }}$ is the Hamiltonian representing the interaction of the radiation field with atom $\xi$. By inspection of equation (2.1) it is immediately apparent, in contrast to most classical descriptions, that energy transfer is not mediated by instantaneous coupling interactions-note the absence of any terms with $\xi^{\prime} \neq \xi$ - but by the quantum field, whose photons exhibit retardation associated with their finite speed of propagation. The eigenstates, $|\mathrm{n}\rangle$, of a basis Hamiltonian given by equation (2.1) excluding $H_{\text {int }}$, form a composite set expressible in the following form:

$$
|\mathrm{n}\rangle=\left|\operatorname{mat}_{\mathrm{n}}\right\rangle\left|\operatorname{rad}_{\mathrm{n}}\right\rangle \equiv\left|\operatorname{mat}_{\mathrm{n}} ; \operatorname{rad}_{\mathrm{n}}\right\rangle \text {. }
$$

Here $\left|\mathrm{mat}_{\mathrm{n}}\right\rangle$ defines the status of all atoms (or molecules, according to the system), comprising a product of state vectors for each atom $\xi$, and $\left|\operatorname{rad}_{n}\right\rangle$ is the radiation (number) state. In the electric-dipole approximation, $H_{\text {int }}(\xi)$ is given by

$$
H_{\text {int }}(\xi)=-\sum_{\xi} \mu(\xi) \cdot \mathbf{e}^{\perp}\left(\mathbf{R}_{\xi}\right) .
$$

In equation (2.3), the electric-dipole moment operator, $\boldsymbol{\mu}(\xi)$, operates on matter states, $\left|\mathrm{mat}_{\mathrm{n}}\right\rangle$, and the transverse electric field operator, $\mathbf{e}^{\perp}\left(\mathbf{R}_{\xi}\right)$, operates on $\left|\operatorname{rad}_{n}\right\rangle$. The latter involves a summation over all wave vectors, $\mathbf{p}$, and polarizations, $\lambda$, and is usually written as the following mode expansion:

$\mathbf{e}^{\perp}\left(\mathbf{R}_{\zeta}\right)=\mathrm{i} \sum_{\mathbf{p}, \lambda}\left(\frac{\hbar c p}{2 \varepsilon_{0} V}\right)^{1 / 2}\left\{\mathbf{e}^{(\lambda)}(\mathbf{p}) a^{(\lambda)}(\mathbf{p}) \mathrm{e}^{\mathrm{i}\left(\mathbf{p} \cdot \mathbf{R}_{\zeta}\right)}-\overline{\mathbf{e}}^{(\lambda)}(\mathbf{p}) a^{\dagger(\lambda)}(\mathbf{p}) \mathrm{e}^{-\mathrm{i}\left(\mathbf{p} \cdot \mathbf{R}_{\zeta}\right)}\right\}$,

where $\mathbf{e}^{(\lambda)}(\mathbf{p})$ is the polarization unit vector $\left(\overline{\mathbf{e}}^{(\lambda)}(\mathbf{p})\right.$ being its complex conjugate), $V$ is an arbitrary quantization volume and $a^{(\lambda)}(\mathbf{p}), a^{\dagger(\lambda)}(p)$ are respectively the photon annihilation and creation operators for a mode $(\mathbf{p}, \lambda)$. The latter operators act on the radiation states through the relations;

and

$$
a^{(\lambda)}(\mathbf{p})|m(\mathbf{p}, \lambda)\rangle=\sqrt{m}|(m-1)(\mathbf{p}, \lambda)\rangle,
$$

$$
a^{\dagger(\lambda)}(\mathbf{p})|m(\mathbf{p}, \lambda)\rangle=\sqrt{m+1}|m+1(\mathbf{p}, \lambda)\rangle,
$$

the appearance of these operators in $H_{\text {int }}$ signifies photon creation and annihilation.

In an RET process involving electric dipole allowed transitions for both donor decay and acceptor excitation, i.e. an E1-E1 coupling system, the initial state $|i\rangle$ may be written as $\left|\mathrm{A}^{\alpha} ; \mathrm{B}^{0} ; 0\right\rangle$ and the final state $|f\rangle$ as $\left|\mathrm{A}^{0} ; \mathrm{B}^{\beta} ; 0\right\rangle$, using the notation of equation (2.2). Here the superscript 0 denotes the ground energy level, along with $\alpha$ and $\beta$ as the excited levels for the donor and acceptor, respectively-and the states of any atoms not engaged in the transfer are factored out. Hence, energy transfer is mediated by coupling to the vacuum radiation field, invoking (a minimum of) one $a^{(\lambda)}(\mathbf{p})$ and also one $a^{\dagger(\lambda)}(\mathbf{p})$ operator, whose two distinct time-orderings correspond to the Feynman diagrams of figures 2(a) and (b). Physically, these two sequences are interpreted as: (a) the creation of a virtual photon at A and its subsequent annihilation at B; (b) vice versa. The virtual photon can be understood as 


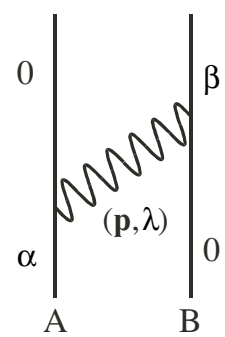

(a)

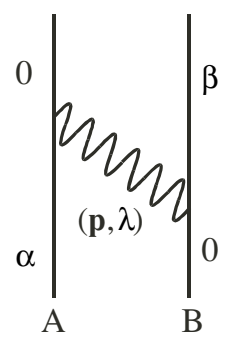

(b)

Figure 2. The two Feynman diagrams for resonance energy transfer with E1-E1 coupling: 0 denotes the ground-state level, $\alpha$ and $\beta$ the excited levels for the donor and acceptor, respectively.

'borrowing' energy from the vacuum, consistent with the energy uncertainty $\hbar / t$, where $t$ is the photon time of flight (here, determined by the distance between the two atoms). This also signifies a temporary relaxation of exact energy conservation in the isolated photon creation and annihilation events. When the whole system enters its final state, i.e. after the virtual photon is extinguished, energy conservation is restored. This is a key feature of virtual photon behaviour; other aspects emerge later.

With two virtual photon-matter interactions and the corresponding $H_{\text {int }}(\xi)$ acting as a perturbation, the quantum amplitude, $M_{f i}^{\mathrm{e}-\mathrm{e}}$, for E1-E1 coupling is calculated from the second term of an expansion in time-dependent perturbation theory;

$$
M_{f i}^{\mathrm{e}-\mathrm{e}}=\frac{\left\langle f\left|H_{\mathrm{int}}\right| r_{a}\right\rangle\left\langle r_{a}\left|H_{\mathrm{int}}\right| i\right\rangle}{\left(E_{i}-E_{r_{a}}\right)}+\frac{\left\langle f\left|H_{\mathrm{int}}\right| r_{b}\right\rangle\left\langle r_{b}\left|H_{\mathrm{int}}\right| i\right\rangle}{\left(E_{i}-E_{r_{b}}\right)},
$$

where the first and second terms correspond to the time-orderings of figures 2(a) and (b), respectively; also $\left|r_{a}\right\rangle$ and $\left|r_{b}\right\rangle$ are intermediate states, and $E$ denotes the energy of a state as signified by the subscript. These energies comprise a sum of radiation and matter parts and, thus, are written as $E_{i}=E_{\alpha}^{\mathrm{A}}+E_{0}^{\mathrm{B}}, E_{r_{a}}=E_{0}^{\mathrm{A}}+E_{0}^{\mathrm{B}}+\hbar c p$ and $E_{r_{b}}=E_{\alpha}^{\mathrm{A}}+E_{\beta}^{\mathrm{B}}+\hbar c p$, where the subscript denotes atom $\xi$ in either the ground or excited state and $\hbar c p$ is the photon energy. Simplification results from use of the overall energy conservation identity;

$$
E_{\alpha 0}^{\mathrm{A}}=E_{\alpha}^{\mathrm{A}}-E_{0}^{\mathrm{A}}=E_{\beta 0}^{\mathrm{B}}=E_{\beta}^{\mathrm{B}}-E_{0}^{\mathrm{B}} \equiv \hbar c k,
$$

here defining $\hbar c k$ as the physically identifiable transferred energy. Hence, equation (2.5) can be rewritten as

$$
\begin{aligned}
M_{f i}^{\mathrm{e}-\mathrm{e}}=\frac{\left\langle\mathrm{A}^{0} ; \mathrm{B}^{\beta} ; 0\left|H_{\text {int }}\right| \mathrm{A}^{0} ; \mathrm{B}^{0} ; 1(\mathbf{p}, \lambda)\right\rangle\left\langle\mathrm{A}^{0} ; \mathrm{B}^{0} ; 1(\mathbf{p}, \lambda)\left|H_{\text {int }}\right| \mathrm{A}^{\alpha} ; \mathrm{B}^{0} ; 0\right\rangle}{\hbar c k-\hbar c p} & \\
& +\frac{\left\langle\mathrm{A}^{0} ; \mathrm{B}^{\beta} ; 0\left|H_{\text {int }}\right| \mathrm{A}^{\alpha} ; \mathrm{B}^{\beta} ; 1(\mathbf{p}, \lambda)\right\rangle\left\langle\mathrm{A}^{\alpha} ; \mathrm{B}^{\beta} ; 1(\mathbf{p}, \lambda)\left|H_{\text {int }}\right| \mathrm{A}^{\alpha} ; \mathrm{B}^{0} ; 0\right\rangle}{-\hbar c k-\hbar c p}
\end{aligned}
$$

By the application of equations (2.3) and (2.4) in (2.7)

$$
M_{f i}^{\mathrm{e}-\mathrm{e}}=\left(2 \varepsilon_{0} V\right)^{-1} \sum_{\mathbf{p}, \lambda} p \bar{e}_{i}^{(\lambda)}(\mathbf{p}) e_{j}^{(\lambda)}(\mathbf{p})\left\{\frac{\mu_{i}^{0 \alpha(\mathrm{A})} \mu_{j}^{\beta 0(\mathrm{~B})} \mathrm{e}^{\mathrm{i}(\mathbf{p} \cdot \mathbf{R})}}{k-p}+\frac{\mu_{j}^{0 \alpha(\mathrm{A})} \mu_{i}^{\beta 0(\mathrm{~B})} \mathrm{e}^{-\mathrm{i}(\mathbf{p} \cdot \mathbf{R})}}{-k-p}\right\} .
$$

Here a concise notation for the transition dipole moments is introduced, e.g. $\boldsymbol{\mu}^{0 \alpha(\mathrm{A})} \equiv$ $\left\langle\mathrm{A}^{0}\left|\boldsymbol{\mu}^{(\mathrm{A})}\right| \mathrm{A}^{\alpha}\right\rangle$, and the convention of summation over repeated Cartesian indices is implemented; also $\mathbf{R}=\mathbf{R}_{\mathrm{B}}-\mathbf{R}_{\mathrm{A}}$ is the interatomic separation vector. The wave-vector and polarization summations in equation (2.8) may be evaluated following the standard techniques of Craig and Thirunamachandran [15]. Extending the boundaries of the quantization volume 
we recognize that each point in $\mathbf{p}$-space represents a realizable $\mathbf{p}$-vector and the wave vector converts to an integral as $V \rightarrow \infty$,

$$
\lim _{V \rightarrow \infty} \frac{1}{V} \sum_{\mathbf{p}} \equiv \int \frac{\mathrm{d}^{3} \mathbf{p}}{(2 \pi)^{3}}
$$

The polarization sum is tackled using the sum rule,

$$
l_{i \alpha} l_{j \alpha}=\delta_{i j},
$$

where $l_{i \alpha}$ is the cosine of the angle between an axis in the laboratory frame (denoted by Roman letters) and one in an independent frame (Greek). Choosing the orthogonal frame set $\mathbf{e}^{(1)}(\mathbf{p})$, $\mathbf{e}^{(2)}(\mathbf{p})$ and $\mathbf{p}$ as the independent frame gives

$$
e_{i}^{(1)}(\mathbf{p}) \bar{e}_{j}^{(1)}(\mathbf{p})+e_{i}^{(2)}(\mathbf{p}) \bar{e}_{j}^{(2)}(\mathbf{p})+\hat{p}_{i} \hat{p}_{j}=\delta_{i j},
$$

so that the polarization sum can be expressed as

$$
\sum_{\lambda} e_{i}^{(\lambda)}(\mathbf{p}) \bar{e}_{j}^{(\lambda)}(\mathbf{p})=\delta_{i j}-\hat{p}_{i} \hat{p}_{j}
$$

Implementing equations (2.9) and (2.12) in (2.8) gives

$$
M_{f i}^{\mathrm{e}-\mathrm{e}}=\frac{\mu_{i}^{0 \alpha(\mathrm{A})} \mu_{j}^{\beta 0(\mathrm{~B})}}{2 \varepsilon_{0}} \int p\left(\delta_{i j}-\hat{p}_{i} \hat{p}_{j}\right)\left\{\frac{\mathrm{e}^{\mathrm{i}(\mathbf{p} \cdot \mathbf{R})}}{k-p}+\frac{\mathrm{e}^{-\mathrm{i}(\mathbf{p} \cdot \mathbf{R})}}{-k-p}\right\} \frac{\mathrm{d}^{3} \mathbf{p}}{(2 \pi)^{3}} .
$$

Next converting to spherical coordinates, $\mathrm{d}^{3} \mathbf{p} \Rightarrow p^{2} \mathrm{~d} \mathbf{p} \mathrm{d} \Omega$, and with

$$
-\int \hat{p}_{i} \hat{p}_{j} \mathrm{e}^{ \pm \mathrm{i}(\mathbf{p} \cdot \mathbf{R})} \mathrm{d} \Omega=\frac{1}{p^{2}} \nabla_{i} \nabla_{j} \int \mathrm{e}^{ \pm \mathrm{i}(\mathbf{p} \cdot \mathbf{R})} \mathrm{d} \Omega,
$$

a change of variables allows (2.13) to be expressed as

$M_{f i}^{\mathrm{e}-\mathrm{e}}=\frac{\mu_{i}^{0 \alpha(\mathrm{A})} \mu_{j}^{\beta 0(\mathrm{~B})}}{4 \pi^{2} \varepsilon_{0}}\left(-\nabla^{2} \delta_{i j}+\nabla_{i} \nabla_{j}\right) \int_{0}^{2 \pi} \int_{-1}^{1} \int_{0}^{\infty} \frac{p}{4 \pi}\left\{\frac{\mathrm{e}^{\mathrm{i}(\mathbf{p} \cdot \mathbf{R})}}{k-p}+\frac{\mathrm{e}^{-\mathrm{i}(\mathbf{p} \cdot \mathbf{R})}}{-k-p}\right\} \mathrm{d} p \mathrm{~d}(\cos \theta) \mathrm{d} \phi$.

Performing the angular integration gives

$$
M_{f i}^{\mathrm{e}-\mathrm{e}}=\frac{\mu_{i}^{0 \alpha(\mathrm{A})} \mu_{j}^{\beta 0(\mathrm{~B})}}{4 \pi^{2} \varepsilon_{0}}\left(-\nabla^{2} \delta_{i j}+\nabla_{i} \nabla_{j}\right) G(k, R),
$$

where the Green function, $G(k, R)$, is defined by

$$
G(k, R)=\int_{-\infty}^{\infty} \frac{\sin p R}{2 R}\left\{\frac{1}{k-p}+\frac{1}{-k-p}\right\} \mathrm{d} p .
$$

Equation (2.17) is evaluated by contour integration to give [16]

$$
G(k, R)=-\frac{\pi}{R} \mathrm{e}^{\mp \mathrm{i} k R}
$$

Hence, inserting equation (2.18) into (2.16), and performing the necessary vector differentiation, produces the following result:

$$
M_{f i}^{\mathrm{e}-\mathrm{e}}=\mu_{i}^{0 \alpha(\mathrm{A})} V_{i j}(k, \mathbf{R}) \mu_{j}^{\beta 0(\mathrm{~B})},
$$

where the E1-E1 coupling tensor, $V_{i j}(k, \mathbf{R})$, is expressed as

$$
V_{i j}(k, \mathbf{R})=\frac{\mathrm{e}^{\mathrm{i} k R}}{4 \pi \varepsilon_{0} R^{3}}\left\{\left(\delta_{i j}-3 \hat{R}_{i} \hat{R}_{j}\right)-(\mathrm{i} k R)\left(\delta_{i j}-3 \hat{R}_{i} \hat{R}_{j}\right)-(k R)^{2}\left(\delta_{i j}-\hat{R}_{i} \hat{R}_{j}\right)\right\} .
$$

This is the key result, to be given detailed examination in the following section 2.2. 
On examination of equation (2.20), it is immediately evident that the first term is dominant in the short-range or near-zone $e^{1}$ region $(k R \ll 1)$, and the third term in the long-range or wave-zone $(k R \gg 1)$. It is of passing interest to note that the electric field, given by $E_{j}=\mu_{i} V_{i j}(k, \mathbf{R})$ in QED, has an equivalent form [17] to the result from classical retarded electrodynamics - which, recast in SI units, is given by $[18]^{2}$

$\mathbf{E}=k^{2}(\hat{\mathbf{R}} \times \boldsymbol{\mu}) \times \hat{\mathbf{R}} \frac{\mathrm{e}^{\mathrm{i} k R}}{4 \pi \varepsilon_{0} R}+[3 \hat{\mathbf{R}}(\hat{\mathbf{R}} \cdot \boldsymbol{\mu})-\boldsymbol{\mu}]\left(\frac{1}{4 \pi \varepsilon_{0} R^{3}}-\frac{\mathrm{i} k}{4 \pi \varepsilon_{0} R^{2}}\right) \mathrm{e}^{\mathrm{i} k R}$.

Note that only the first term in (2.21), corresponding to the third term of equation (2.20), is necessarily transverse w.r.t. R.

\subsection{Properties and physical interpretation}

It is possible to elicit several useful physical insights, including some detailed aspects of the electromagnetic coupling, from the quantum amplitude for E1-E1 coupling-as determined by the structure of $M_{f i}^{\mathrm{e}-\mathrm{e}}(2.19)$ and the corresponding $V_{i j}(k, \mathbf{R})$ tensor for dynamic (transition) and static (permanent) electric dipoles.

2.2.1. Quantum amplitude for dynamic coupling. In dynamic E1-E1 coupling, a number of important features can be identified by simple manipulations on equation (2.20) as follows:

(i) Taking the product of (2.20) with $R_{i} R_{j}$ (with implied summation over $i$ and $j$ ) it is apparent that the third (or long range) term disappears, so that

$$
V_{i j} R_{i} R_{j}=0 \text { (long range). }
$$

Thus, by using simple dot product rules, it emerges that the $\mathbf{V}$ tensor is orthogonal to an outer product of $\mathbf{R}$ vectors and, therefore, the coupling tensor is fully transverse w.r.t. $\mathbf{R}$ at large distances-i.e. for $\mathbf{R} \gg \lambda$, where $\lambda=2 \pi / k$ designates the wavelength regime associated with the energy being transferred.

(ii) In comparison, the full result of contracting $V_{i j}$ with $R_{i} R_{j}$ includes both the remaining terms of (2.20), as the following shows:

$$
V_{i j} R_{i} R_{j}=\frac{\mathrm{e}^{\mathrm{i} k R}}{4 \pi \varepsilon_{0} R^{3}}\{-2(1-\mathrm{i} k R)\} .
$$

Therefore, for the shorter-range terms, the coupling tensor is not fully transverse w.r.t. $\mathbf{R}$ - and hence contains a longitudinal component.

(iii) Due to the presence of the first term of equation (2.20), deployed within the quantum amplitude of equation (2.19), it is always possible for $M_{f i}^{\mathrm{e}-\mathrm{e}}$ to be nonzero for $\boldsymbol{\mu}^{\mathrm{A}} \perp \boldsymbol{\mu}^{\mathrm{B}}$. In fact the only case where the coupling necessarily vanishes, given the condition $\boldsymbol{\mu}^{\mathrm{A}} \perp \boldsymbol{\mu}^{\mathrm{B}}$, is when one of these transition dipoles is also orthogonal to $\mathbf{R}$ - and in this situation all three terms of equation (2.20), cast in (2.19), equal zero.

Physically, it is apparent that all three terms of equation (2.20) are required when considering dynamic E1-E1 coupling. Despite this, the term involved in (i) is dominant in the long-range region and indicates a highly 'real' character of the electromagnetic mediatorthis explains why 'radiative' energy transfer is commonly associated with interacting dynamic dipoles in the long range. In detail, the transversality of the coupling field w.r.t. $\mathbf{R}$ is consistent

1 Elsewhere, the term near-field $[19,20]$ often relates to evanescent waves and concerns local transmission at an interface [21].

2 This equation—and also (3.10)—differs from Jackson by the following symbol substitutions; $\mathbf{n} \rightarrow \hat{\mathbf{R}}, \mathbf{p} \rightarrow \boldsymbol{\mu}$ and $r \rightarrow R$. 


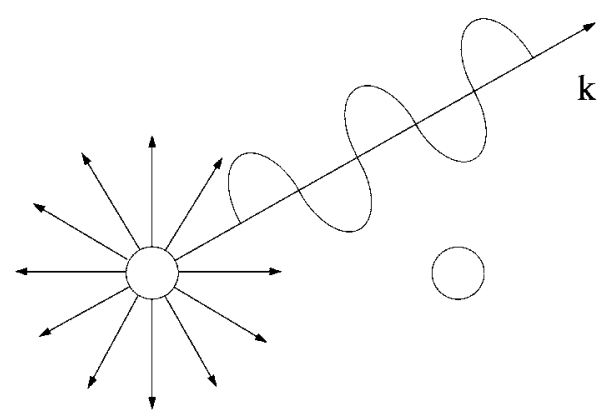

(a)

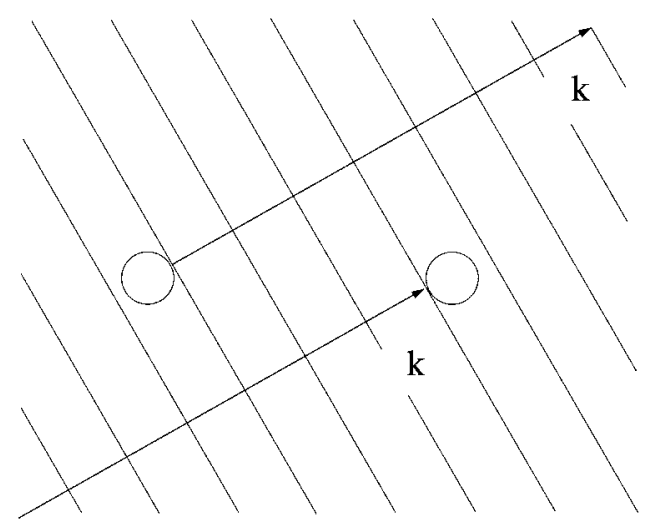

(b)

Figure 3. (a) Virtual photon emission from the donor, accommodating modes with wave-vectors p pointing in all directions. Donor $\mathrm{A}$ is on the left, acceptor $\mathrm{B}$ on the right. Focusing on one particular mode with wave-vector $\mathbf{p}=\mathbf{k}$; (b) shows the associated wave fronts (diagonal lines). The two differently positioned vectors $\mathbf{k}$ signify the same electromagnetic mode; hence a virtual photon may be emitted by the donor in a direction that does not coincide with the interatomic separation vector and still be absorbed by the acceptor.

with the fact that photon fields are always transverse w.r.t. their wave-vector $\mathbf{p}$, and only photons with $\mathbf{p}$ essentially collinear to $\mathbf{R}$ remain significant as the interatomic separation increases towards infinity. In contrast, (ii) evinces a longitudinal component w.r.t. $\mathbf{R}$ in the short-range region, which derives from the fact that photons with $\mathbf{p}$ not parallel to $\mathbf{R}$ are also involved in the energy transfer. This is consistent with the position-momentum uncertainty principle, i.e. the positions of the photon creation and annihilation events are uncertain. See, for example, figure 3 which shows: (a) virtual photon emission from the donor in all directions, with the focus on one particular electromagnetic mode; (b) this same mode in two differing localizations.

Thus the effect of reducing, from the long range, the interatomic separation is to increase the virtual character of the mediator. In the short-range limit $(R \rightarrow 0)$ significant retardation is absent and the virtual nature of the photon accords with an understanding reflected by the widespread adoption of the term 'radiationless' energy transfer. Finally, (iii) highlights the possible occurrence of E1-E1 energy transfer even if $\boldsymbol{\mu}^{\mathrm{A}}$ is orthogonal to $\boldsymbol{\mu}^{\mathrm{B}}$ - despite occasional assertions to the contrary $[22,23]$-though, for example, an orthogonal triad of $\boldsymbol{\mu}^{\mathrm{A}}, \boldsymbol{\mu}^{\mathrm{B}}$ and $\mathbf{R}$ does indeed preclude coupling of this type. 
2.2.2. Static dipole coupling. In the special case where static E1-E1 coupling occurs, it is of interest to note the following features:

(iv) An equivalent expression to that derived classically from Coulomb's law, i.e. a representation of two interacting static electric dipoles, is found when $k=0$ in equation (2.19). This may be written as [15]

$\lim _{k \rightarrow 0} M_{f i}^{\mathrm{e}-\mathrm{e}}=\frac{\mu_{i}^{\mathrm{A}} \mu_{j}^{\mathrm{B}}}{4 \pi \varepsilon_{0} R^{3}}\left(\delta_{i j}-3 \hat{R}_{i} \hat{R}_{j}\right)=\frac{1}{4 \pi \varepsilon_{0} R^{3}}\left\{\boldsymbol{\mu}^{\mathrm{A}} \cdot \boldsymbol{\mu}^{\mathrm{B}}-3\left(\boldsymbol{\mu}^{\mathrm{A}} \cdot \hat{\mathbf{R}}\right)\left(\boldsymbol{\mu}^{\mathrm{B}} \cdot \hat{\mathbf{R}}\right)\right\}$,

with the shorthand notation $\boldsymbol{\mu}^{0 \alpha(\mathrm{A})} \equiv \boldsymbol{\mu}^{\mathrm{A}}$ and $\boldsymbol{\mu}^{\beta 0(\mathrm{~B})} \equiv \boldsymbol{\mu}^{\mathrm{B}}$.

(v) Due to nonzero results for both $\mu^{\xi} \perp \mathbf{R}$ and $\mu^{\xi} \| \mathbf{R}$ in equation (2.24), the coupling tensor in the interaction of two static electric dipoles has both longitudinal and transverse components w.r.t. $\mathbf{R}$.

(vi) The quantum amplitude for dynamic E1-E1 coupling at the short-range limit converges to the expression of equation (2.24).

The physical insights acquired from these last features are: equation (2.24), as the quantum amplitude corresponding to the first (or short-range) term of (2.20), also represents the coupling energy of two static electric dipoles in accordance with the classical expression; from (v) it is apparent that the coupling has both longitudinal and transverse components w.r.t. $\mathbf{R}$, i.e. the fields are virtual in character. In fact, for static electric dipoles to be the source of dynamic fields, the latter must be associated with energy derived from vacuum 'borrowing'; lastly, (vi) supports the conclusion that dynamic E1-E1 coupling in the short-range limit has virtual field character.

2.2.3. Transfer rate for dipole coupling. Let us now focus attention on the transfer rate, $\Gamma$, which represents a direct physical observable derived from the $V_{i j}$ tensor, and which provides a basis for the interpretation of further properties of the system. To determine the rate of transfer with E1-E1 coupling we use Fermi's golden rule [24], to give

$$
\Gamma=\frac{2 \pi}{\hbar}\left|M_{f i}^{\mathrm{e}-\mathrm{e}}\right|^{2} \rho_{f},
$$

where $\rho_{f}$ is the density of acceptor final states. For a system of two freely rotating dipoles equation (2.25) also requires the implementation of rotational averaging [25], and delivers a result expressible as

$$
\Gamma \sim \frac{1}{9}\left|\boldsymbol{\mu}^{\mathrm{A}}\right|^{2}\left|\boldsymbol{\mu}^{\mathrm{B}}\right|^{2} A(k, R),
$$

where the excitation transfer function, $A(k, R)$, is defined by [26]

$$
A(k, R)=V_{i j}(k, \mathbf{R}) \bar{V}_{i j}(k, \mathbf{R})=\frac{2}{\left(4 \pi \varepsilon_{0} R^{3}\right)^{2}}\left\{3+(k R)^{2}+(k R)^{4}\right\} .
$$

The result, (2.27), signifies a scalar characterizing the E1-E1 transfer rate for any specific energy and separation. From this equation the following points are noteworthy:

(vii) The initial and final terms dominate in the short- and long-range regions, respectively, as follows from the corresponding terms in equation (2.20). This is consistent with the $R^{-6}$ dependence of Förster's result [27] for 'radiationless' energy transfer and the $R^{-2}$ dependence of classical inverse square laws for 'radiative' transfer. The presence of these terms (and the distinctive middle term) in a single expression signifies that these two processes are the short- and long-range asymptotes of a single mechanism accommodated in a unified theory [26]. 


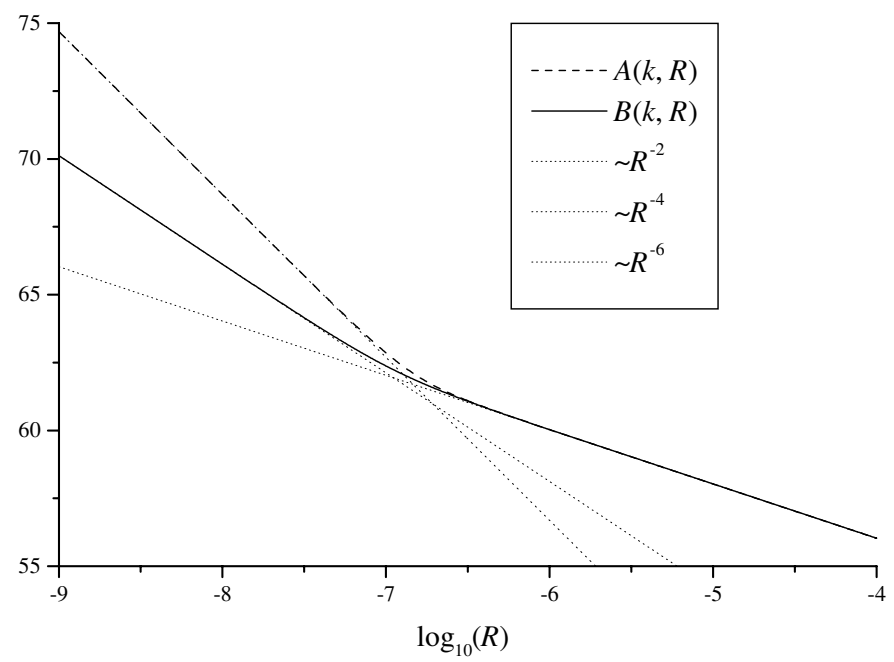

Figure 4. $\log -\log$ plot of the transfer functions $A(k, R)$ and $B(k, R)$ against distance $R$ (in $\mathrm{m}$ ), over appropriate transfer distances: $k=9 \times 10^{6} \mathrm{~m}^{-1}$.

(viii) The initial and final terms of (2.27) are associated with a mediator of fully virtual and 'real' characteristics, respectively. Physically, neither of these terms can be considered in isolation for dynamic E1-E1 coupling; as described earlier, every photon in principle exhibits both virtual and 'real' traits. Specifically, as dynamic dipoles interact over an increasing distance, the energy transfer exhibits an increasingly 'radiative', propagating behaviour - though a partly virtual character always remains. Equally, at small distances, what is normally termed 'radiationless' energy transfer retains some attributes of 'real' photon exchange.

(ix) The graph of figure 4 shows a $\log -\log$ plot of the $A(k, R)$ function response over the range $1 \mathrm{~nm}$ to $1 \mu \mathrm{m}$, for a value of $k=9 \times 10^{6} \mathrm{~m}^{-1}$. This figure gives an excellent representation of the Förster behaviour and 'radiative' transfer rate as short- and longrange asymptotes, respectively, shown by the variation in gradient between the shortand long-range regions.

It is evident that the QED formulation offers a range of immediate and accessible insights into the physics of transition dipole coupling. In the following section, we extend this approach to include magnetic dipole effects.

\section{E1-M1 coupling}

\subsection{Derivation of the coupling tensor}

The basis of resonance energy transfer in the previous section was a coupling of two electric dipoles, and in most cases dipolar coupling of this form is indeed primarily responsible for energy transfer. However if, for example, either the donor decay or the acceptor excitation transition is electric dipole forbidden, or the geometry precludes E1-E1 coupling, then atomic coupling between electric and magnetic dipoles may become the dominant mechanism for RET. To derive an E1-M1 coupling tensor requires the same method as that of section 2, but 


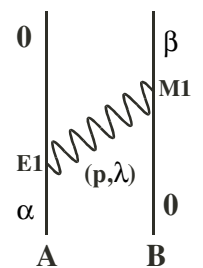

(a)

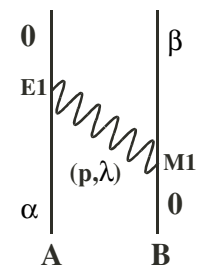

(b)

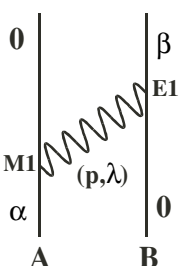

(c)

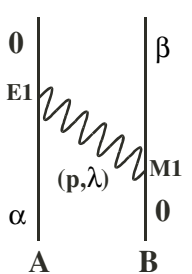

(d)

Figure 5. The four Feynman diagrams for resonance energy transfer with E1-M1 coupling: E1 and M1 denote electric and magnetic dipole interactions, respectively.

the interaction Hamiltonian of equation (2.3) is expanded to accommodate interactions of both electric and magnetic dipoles. Therefore, (2.3) becomes

$$
H_{\text {int }}=-\sum_{\xi=\mathrm{A}, \mathrm{B}}\left\{\boldsymbol{\mu}(\xi) \cdot \mathbf{e}^{\perp}\left(\mathbf{R}_{\xi}\right)+\mathbf{m}(\xi) \cdot \mathbf{b}\left(\mathbf{R}_{\xi}\right)\right\}
$$

exhibiting both the magnetic dipole operator, $\mathbf{m}(\xi)$, and the magnetic field operator, $\mathbf{b}\left(\mathbf{R}_{\xi}\right)$. The latter operator may again be expressed as a mode expansion— $\mathrm{cf}(2.4)$;

$\mathbf{b}\left(\mathbf{R}_{\xi}\right)=\mathrm{i} \sum_{\mathbf{p}, \lambda}\left(\frac{\hbar k}{2 \varepsilon_{0} c V}\right)^{\frac{1}{2}}\left\{\mathbf{b}^{(\lambda)}(\mathbf{p}) a^{(\lambda)}(\mathbf{p}) \mathrm{e}^{\mathrm{i} \cdot \mathbf{R}_{\xi}}-\overline{\mathbf{b}}^{(\lambda)}(\mathbf{p}) a^{\dagger(\lambda)}(\mathbf{p}) \mathrm{e}^{-\mathrm{i} \cdot \mathbf{\mathbf { R } _ { \xi }}}\right\}$,

where $\mathbf{b}^{(\lambda)}(\mathbf{p})=\hat{\mathbf{p}} \times \mathbf{e}^{(\lambda)}(\mathbf{p})$ is the magnetic polarization vector in the direction of the magnetic field vector, and $\overline{\mathbf{b}}^{(\lambda)}(\mathbf{p})$ is its complex conjugate. The incorporation of magnetic interactions into $H_{\text {int }}$ increases the number of contributions to the quantum amplitude of RET and the Feynman diagrams of figures 2(a) and (b) are conveniently modified to accommodate this change, as shown in figures 5(a)-(d). With E1-M1 coupling, account can be taken of electric and magnetic dipole interactions for the donor decay and acceptor excitation, respectively, and also the converse, i.e. a magnetic dipole allowed decay transition and an electric dipole allowed excitation. Hence the quantum amplitude for E1-M1 coupling is composed of two parts, $M_{f i}^{\mathrm{e}-\mathrm{m}}+M_{f i}^{\mathrm{m}-\mathrm{e}}$, where the superscript pair denotes the transition dipoles for A and B respectively. Applying similar methods to those employed in the previous section, the result emerges explicitly as follows:

$M_{f i}^{\mathrm{e}-\mathrm{m}}+M_{f i}^{\mathrm{m}-\mathrm{e}}=\frac{-\mathrm{i} k}{2 \pi^{2} \varepsilon_{0} c} \varepsilon_{i j k} \hat{R}_{k}\left\{\mu_{i}^{0 \alpha(\mathrm{A})} m_{j}^{\beta 0(\mathrm{~B})}+m_{j}^{0 \alpha(\mathrm{A})} \mu_{i}^{\beta 0(\mathrm{~B})}\right\} G^{\prime}(k, R)$,

assuming all these transition dipoles are allowed. In equation (3.3) the Green function, $G^{\prime}(k, R)$, is written as

$$
G^{\prime}(k, R)=\int_{0}^{\infty} \frac{1}{k^{2}-p^{2}}\left(\frac{p^{2} \cos p R}{R}-\frac{p \sin p R}{R^{2}}\right) \mathrm{d} p .
$$

The derivation of (3.3) utilizes a polarization sum cast in terms of $\varepsilon_{i j k}$, the third-rank, antisymmetric Levi-Civita tensor,

$$
\sum_{\lambda} e_{i}^{(\lambda)}(\mathbf{p}) \bar{b}_{l}^{(\lambda)}(\mathbf{p})=\varepsilon_{j k l} \hat{p}_{k}\left(\delta_{i l}-\hat{p}_{i} \hat{p}_{l}\right)=\varepsilon_{i j k} \hat{p}_{k},
$$

as follows from $\overline{\mathbf{b}}^{(\lambda)}(\mathbf{p})=\hat{\mathbf{p}} \times \overline{\mathbf{e}}^{(\lambda)}(\mathbf{p})$ and $\varepsilon_{j k l} \hat{p}_{k} \hat{p}_{l}=\hat{\mathbf{p}} \times \hat{\mathbf{p}}=0$. Also used is the identity;

$$
\frac{1}{4 \pi} \int \hat{p}_{k} \mathrm{e}^{ \pm \mathrm{i} \cdot \mathbf{R}} \mathrm{d} \Omega=\mp \mathrm{i}\left(\frac{\cos p R}{p R}-\frac{\sin p R}{p^{2} R^{2}}\right) \hat{R}_{k},
$$


giving, after performing the necessary contour integrations;

$$
M_{f i}^{\mathrm{e}-\mathrm{m}}+M_{f i}^{\mathrm{m}-\mathrm{e}}=\left\{\mu_{i}^{0 \alpha(\mathrm{A})} \frac{m_{j}^{\beta 0(\mathrm{~B})}}{c}+\frac{m_{j}^{0 \alpha(\mathrm{A})}}{c} \mu_{i}^{\beta 0(\mathrm{~B})}\right\} U_{i j}(k, \mathbf{R}) .
$$

The result features the fully retarded E1-M1 coupling tensor, $U_{i j}(k, \mathbf{R})$, expressed as [16]

$$
U_{i j}(k, \mathbf{R})=\frac{\mathrm{e}^{-\mathrm{i} k R}}{4 \pi \varepsilon_{0}} \varepsilon_{i j k} \frac{\hat{R}_{k}}{R^{3}}\left(-\mathrm{i} k R+k^{2} R^{2}\right) .
$$

The first and second terms of (3.8) dominate in the short- and long-range regions, respectively.

It is of note that the magnetic field deriving from electric dipole emission, given by $B_{j}=\mu_{i} U_{i j}(k, \mathbf{R})$ in QED, has an equivalent expression [17] to that of classical electrodynamics [18],

$$
\mathbf{B}=k^{2}(\hat{\mathbf{R}} \times \boldsymbol{\mu}) \frac{\mathrm{e}^{\mathrm{i} k R}}{4 \pi \varepsilon_{0} c R}\left(1-\frac{1}{\mathrm{i} k R}\right) .
$$

By contrast to the $\mathbf{E}$ field in the E1-E1 case, equation (2.21), this $\mathbf{B}$ field is entirely transverse w.r.t. $\mathbf{R}$, at all distances, although it has terms in both $R^{-1}$ and $R^{-2}$. This shows that there can in some such cases be a distinction between fields that are purely propagating (i.e. run with $R^{-1}$ as real photons) and those that are fully transverse with respect to $\mathbf{R}$; the two concepts do not have a 1:1 mapping.

\subsection{Properties and physical interpretation}

As in the previous section, significant physical insights are afforded by study of the quantum amplitude and especially, in this case, the $U_{i j}(k, \mathbf{R})$ tensor.

3.2.1. Quantum amplitude for dynamic coupling. By examination of equations (3.7) and (3.8), a number of noteworthy points are readily established:

(i) First, for clarity equation (3.7) may be rewritten in the form

$$
M_{f i}^{\mathrm{e}-\mathrm{m}}+M_{f i}^{\mathrm{m}-\mathrm{e}}=\frac{\mathrm{e}^{-\mathrm{i} k R}\left(-\mathrm{i} k R+k^{2} R^{2}\right)}{4 \pi c \varepsilon_{0} R^{3}}\left\{\hat{\mathbf{R}} \cdot\left(\boldsymbol{\mu}^{\mathrm{A}} \times \mathbf{m}^{\mathrm{B}}\right)+\left(\mathbf{m}^{\mathrm{A}} \times \boldsymbol{\mu}^{\mathrm{B}}\right) \cdot \hat{\mathbf{R}}\right\},
$$

as follows from $\varepsilon_{i j k} \mu_{i} m_{j} \hat{R}_{k} \equiv(\boldsymbol{\mu} \times \mathbf{m}) \cdot \hat{\mathbf{R}}$ with the introduction of shorthand notation. Hence, it is apparent that the quantum amplitude of equation (3.10) is exactly zero for $\boldsymbol{\mu}^{\mathrm{A}} \| \mathbf{m}^{\mathrm{B}}$ and $\mathbf{m}^{\mathrm{A}} \| \boldsymbol{\mu}^{\mathrm{B}}$. Equally, if either dipole is aligned parallel with the unit vector $\hat{\mathbf{R}}$, a null result is again obtained.

(ii) In the comparison of equations (2.20) and (3.8), i.e. the tensors for E1-E1 and E1-M1 coupling, respectively, a major difference in the latter is the absence of an $R^{-3}$ term. Thus, the two coupling tensors have significantly different $R$ dependence in the shortrange region.

The physical insight acquired from (i) is that parallel electric and magnetic dipoles do not couple. Although an oversimplification, this is consistent with an interpretation based on the alignment of each dynamic dipole with either the electric or magnetic component of the coupling field modes - such that one or other dipole is unable to interact with the same photon. Certainly, for e-m and m-e transfer, a parallel alignment of either the donor or the acceptor transition dipole with the unit vector $\hat{\mathbf{R}}$ demolishes the corresponding coupling. As transpires from (ii), the short-range term is missing in the E1-M1 coupling tensor and, consequently, the electromagnetic mediator of this process has an at least partially propagating character, i.e. a photon that is never fully virtual in nature. 


\subsubsection{Static dipole coupling}

(iii) The absence of the $R^{-3}$ term in equation (3.8), in contrast to the static E1-E1 coupling expression of (2.24), gives a clear indication that (3.8) will give a null result when $k$ is set to zero. Hence, the static E1-M1 coupling equation is given as follows:

$$
\lim _{k \rightarrow 0}\left(M_{f i}^{\mathrm{e}-\mathrm{m}}+M_{f i}^{\mathrm{m}-\mathrm{e}}\right)=0 .
$$

From (iii) it is clear that static E1-M1 coupling does not exist.

3.2.3. Transfer rate of dipole coupling. Once again, further properties emerge from analysis of the transfer rate, $\Gamma^{\prime}$, for the E1-M1 system. Equation (2.25) is rewritten to give the rate as follows:

$$
\Gamma^{\prime}=\frac{2 \pi}{\hbar}\left|M_{f i}^{\mathrm{e}-\mathrm{m}}+M_{f i}^{\mathrm{m}-\mathrm{e}}\right|^{2} \rho_{f} .
$$

This is given explicitly, again after rotational averaging, as

$$
\Gamma^{\prime} \sim \frac{B(k, R)}{9 c^{2}}\left\{\left|\boldsymbol{\mu}^{\mathrm{A}}\right|^{2}\left|\mathbf{m}^{\mathrm{B}}\right|^{2}+\left|\boldsymbol{\mu}^{\mathrm{B}}\right|^{2}\left|\mathbf{m}^{\mathrm{A}}\right|^{2}-2 \operatorname{Re}\left|\boldsymbol{\mu}^{\mathrm{A}} \cdot \overline{\mathbf{m}}^{\mathrm{A}}\right|\left|\overline{\boldsymbol{\mu}}^{\mathrm{B}} \cdot \mathbf{m}^{\mathrm{B}}\right|\right\},
$$

where the e-m excitation transfer function $B(k, R)$, a scalar characterizing the E1-M1 transfer rate for any specific energy and separation, is written as

$$
B(k, R)=U_{i j}(\mathbf{k}, \mathbf{R}) \bar{U}_{i j}(\mathbf{k}, \mathbf{R})=\frac{2}{\left(4 \pi \varepsilon_{0} R^{3}\right)^{2}}\left(k^{2} R^{2}+k^{4} R^{4}\right) .
$$

The properties of E1-M1 coupling revealed on inspection of equation (3.14) are as follows:

(iv) The $B(k, R)$ function contains no term corresponding to the initial term of the analogous $A(k, R)$ function, i.e. the $R^{-6}$ dependence of E1-E1 'radiationless' energy transfer is not found in the interaction of electric and magnetic dipoles. This is consistent with the absence of an $R^{-3}$ term in the E1-M1 coupling tensor.

(v) In contrast to the above, the long-range (final) terms of the $A(k, R)$ and $B(k, R)$ functions do correspond in functional dependence; both exhibit the $R^{-2}$ dependence of 'radiative' energy transfer. Physically, in the E1-M1 case as in E1-E1 coupling, this term cannot be considered in isolation, as an $R^{-4}$ term also exists. Therefore, E1-M1 dynamic coupling does not involve exclusively 'radiative' energy transfer through the propagation of photons of unequivocally 'real' character — contrary to a commonly held view—as the $R^{-4}$ term represents coupling with partly real and partly virtual characteristics. Nonetheless, E1M1 coupling will be essentially real, especially in the long-range region.

(vi) The graph of figure 4 illustrates the $B(k, R)$ function response over the range $1 \mathrm{~nm}$ to $1 \mu \mathrm{m}$, again for a value of $k=9 \times 10^{6} \mathrm{~m}^{-1}$. This shows $R^{-4}$ behaviour (analogous to the intermediate term of the $A(k, R)$ function) as the short-range asymptote and once again the classical 'radiative' energy transfer as the long-range asymptote.

Although we do not give details here, it is gratifying to note that the physical picture developed above from analysis of E1-E1 coupling applies with equal force to the case of M1-M1 coupling-although contributions involving magnetic dipole transitions both in the donor and the acceptor give negligible amplitude contributions and can usually be discarded. Accordingly, if the analysis is performed the resultant equations are again cast directly in terms of $V_{i j}(k, \mathbf{R})$ and $A(k, R)$. This in turn serves as a reminder of the extensive parallelism of electric and magnetic features in electromagnetism - a parallel that can exemplify and ground principles learned in the broader context of courses on special relativity [27]. 


\section{Discussion}

It is hoped that the analysis we have presented accomplishes a number of objectives. First, it clarifies the fact that the commonly termed radiative and radiationless energy transfer processes are in fact the short- and long-range asymptotes of a single unified mechanism; they are not independent and competing processes as viewed in some literature [28-34]. Hopefully, increasing cognizance of this will begin to be reflected in the way the subjects are taught. Our second aim is to set straight the 'differences' between real and virtual photons. Though it remains less well known than one might hope, the illusory (or at best, largely semantic) nature of this difference has been pointed out by many - for example in one of the standard textbooks on elementary particle physics we find 'In a sense every photon is virtual, being emitted and then sooner or later being absorbed' [35]. In connection with resonance energy transfer our paper gives detailed theoretical support to this principle through the following observations: (i) due to the position-momentum uncertainty principle there will always be an uncertainty $\hbar / R$ in the photon momentum component along the interatomic displacement, even given a large value of $R$. Hence the acceptor can experience electromagnetic fields with wave-vectors $\mathbf{p}$ not necessarily parallel to $\mathbf{R}$, and a longitudinal component w.r.t. $\mathbf{R}$ always exists, i.e. the electric field associated with dynamic coupling is never fully transverse w.r.t. R; (ii) equally, from inspection of the $A(k, R)$ (E1-E1) transfer function of equation (2.27) it is clear that the final 'inverse square' term-usually associated with entirely radiative characteristics—cannot be considered in isolation, and hence in this system the coupling photons are never fully real; (iii) equally, the final term in the $B(k, R)$ (E1-M1) function of equation (3.14) associated with 'real' photons, cannot be considered in isolation and always retains some virtual characteristics. The lessons are clear, and the supporting photophysics affords amenable access to observation of the interplay of quantum theory, electromagnetism and the principles of retardation. As a stand-free vehicle to cultivate a mastery of those principles, the QED analysis of resonance energy transfer proves exemplary in every respect.

\section{Acknowledgments}

DLA and DSB would like to thank the Engineering and Physical Sciences Research Council for funding this work.

\section{References}

[1] Renger T, May V and Kühn O 2001 Phys. Rep. 343137

[2] Cogdell R J, Isaacs N W, Freer A A, Howard T D, Gardiner A T, Prince S M and Papiz M Z 2003 FEBS Lett. 55535

[3] Adronov A and Fréchet J M J 2000 Chem. Commun. 1701

[4] Guldi D M 2002 Chem. Soc. Rev. 3122

[5] dos Remedios C G and Moens P D J 1999 Resonance Energy Transfer ed D L Andrews and A A Demidov (Chichester: Wiley) pp 1-64

[6] MacColl R 2004 Biochim. Biophys. Acta 165773

[7] Ovsyankin V V 1987 Spectroscopy of Solids Containing Rare Earth Ions ed A A Kaplyanskii and R M Macfarlane (Amsterdam: Elsevier) pp 343-481

[8] Oskam K D, Wegh R T, Donker H, van Loef E V D and Meijerink A 2000 J. Alloys Compounds 300-301 421

[9] Jefimenko O D 2004 Eur. J. Phys. 25287

[10] Harpaz A 2002 Eur. J. Phys. 23263

[11] Dushek O and Kuzmin S V 2004 Eur. J. Phys. 25343

[12] Erber T and Latal H G 2003 Eur. J. Phys. 2467

[13] Sekatskii S K and Dietler G 2001 Anal. Biochem. 299263

[14] Power E A 2001 Eur. J. Phys. 22453 
[15] Craig D P and Thirunamachandran T 1998 Molecular Quantum Electrodynamics (Mineola, NY: Dover) pp 142-82

[16] Daniels G J, Jenkins R D, Bradshaw D S and Andrews D L 2003 J. Chem. Phys. 1192264

[17] Power E A and Thirunamachandran T 1983 Phys. Rev. A 282663

[18] Jackson J D 1998 Classical Electrodynamics 3rd edn (New York: Wiley) p 411

[19] Andrew P and Barnes W L 2000 Science 290785

[20] Hellmuth T 2004 Microscopy The Optics Encyclopaedia ed Th G Brown, K Creath, H Kogelnik, M A Kriss, J Schmit and M J Weber (Weinheim: Wiley-VCH) pp 1469-531

[21] Ford G W 2004 Electromagnetic radiation: emission and propagation The Optics Encyclopaedia ed Th G Brown, K Creath, H Kogelnik, M A Kriss, J Schmit and M J Weber (Weinheim: Wiley-VCH) pp 423-77

[22] Haugland R P 2002 Handbook of Fluorescent Probes and Research Products 9th edn (Eugene, OR: Molecular Probes) p 25

[23] http://www.sci.sdsu.edu/TFrey/Bio750/Fluorescence.html

[24] Fermi E 1950 Nuclear Physics (Chicago: University of Chicago Press)

[25] Andrews D L and Thirunamachandran T 1977 J. Chem. Phys. 675026

[26] Andrews D L 1989 Chem. Phys. 135195

[27] Jackson J D 2004 The Optics Encyclopedia. Basic Foundations and Practical Applications vol 1 ed Th G Brown, K Creath, H Kogelnik, M A Kriss, J Schmit and M J Weber (Weinheim: Wiley-VCH) p 416

[28] Förster T 1948 Ann. Phys. 255

[29] Birks J B 1970 Photophysics of Aromatic Molecules (London: Wiley) pp 518-21

[30] Van Der Meer B W, Coker G III and Simon Chen S-Y 1994 Resonance Energy Transfer Theory and Data (New York: VCH) pp 9-10

[31] Guptasarma P and Raman B 1995 Anal. Biochem. 230187

[32] Lakowicz J R 1999 Principles of Fluorescence Spectroscopy 2nd edn (New York: Kluwer Academic/Plenum) p 368

[33] Finlayson C E, Ginger D S and Greenham N C 2001 Chem. Phys. Lett. 33883

[34] Valeur B 2002 Molecular Fluorescence (Weinheim: Wiley-VCH) pp 110-24

[35] Halzen F and Martin A D 1984 Quarks and Leptons (New York: Wiley) p 140 